\title{
Performance of Twin-Fluid Atomizers for Atomization of Viscous Solutions
}

\author{
Marek Mlkvik ${ }^{1, a}$, Philipp Stähle ${ }^{2}$, Volker Gaukel ${ }^{2}$, Matouš Zaremba ${ }^{1}$, Jan Jedelský ${ }^{1}$ and Miroslav Jícha ${ }^{1}$ \\ ${ }^{I}$ Brno University of Technology, Faculty of mechanical engineering, Department of the thermodynamics and \\ environmental engineering, Technická 2896/2, 61669 Brno, Czech Republic \\ ${ }^{2}$ Karlsruhe Institute of Technology, Institute of Process Engineering in Life Sciences, Section I: Food Process \\ Engineering, Kaiserstraße 12, 76131 Karlsruhe, Germany
}

\begin{abstract}
Presented paper deals with a comparison of two internally mixing twin fluid atomizers. The well known Y- jet atomizer and so called outside-in-liquid effervescent atomizer (OUIL) were investigated. The working regimes were defined by the pressure drop $(\Delta p)$ and the gas to the liquid ratio (GLR). The internal and the external two-phase flows of both atomizers were studied. The influence of the mixing mechanism on the internal flow was evaluated by the gas to the liquid momentum ratio $(\Phi)$. In advance, the stability of the separated flow (liquid film) was examined in term of the critical wavelength of the surface disturbances $\left(\lambda_{c}\right)$. The external flow was observed by the high-speed camera. The influence of the basic forces on the deformation of the liquid was determined by a dimensionless criterion $w \cdot \mu / \sigma$. The values of $\Phi<1$ for the OUIL atomizer indicates that the internal flow of this atomizer was annular. The internal flow Y-jet atomizer was more complicated, because the momentum ratio varies from $\Phi<1$, where the gas momentum is dominant, up to $\Phi>3$, where the liquid momentum overcomes the gas momentum. The values of $w \cdot \mu / \sigma>20$ for both atomizers indicates the dominant influence of the viscosity and the drag force on the breakup process.
\end{abstract}

\section{Introduction}

The pneumatic nozzles are commonly used to atomize the viscous liquids in many industrial applications. They produce high shear stresses originating from a high gas to liquid relative velocity, which supports the atomization process [1]. Several types of the internal mixing atomizers, were presented in $[7,8]$. The internal flow is characterized by the mixing of the flow components, motion and deformation of the liquid and the gas structures, as the result of the ratio of the forces such as the drag force, the viscous forces $(\mu)$ and the surface tension $(\sigma)$. The development of the multiphase flow is also accompanied with the energy conversion $[2,5]$. The flow pattern of the two - phase flow often depends on the GLR which is related to the mass flow of the mixture components, or to the void fraction $(\alpha)$ which corresponds to the volume of the particular phase. Also the mixing mechanism influences the flow pattern [7, 2]. After passing the output orifice, the gas expands due to the pressure drop, which leads to the increase of interfacial surface, as the liquid membranes and the ligaments are created [9]. Presented work compares two types of internally mixing twin fluid atomizers, the Y-jet atomizer [7] and so called "outside in liquid" (OUIL) atomizer which is not used in industrial applications yet. The internal flow is evaluated using the dimensionless numbers, such as GLR and the liquid to the gas momentum ratio $(\Phi)$, which is calculated according to Song [8]. The external flow is investigated by the visualization and evaluated in terms of dimensionless numbers to determine the relative influence of the basic forces on the liquid during the atomization process.

\section{Atomizers}

The multiphase flow in the $\mathrm{Y}$-jet is created by injecting of the liquid into the air stream radially, under a particular angle to the axis (Figure 1 a). The two-phase flow is created inside the mixing chamber and it disintegrates when it reaches the output orifice. In the OUIL atomizer (Figure $1 \mathrm{~b}$ ), the air is introduced to the mixing chamber from the top and the liquid is introduced through the series of liquid ports in the perforated wall of the mixing chamber. Although the design of the two examined atomizers is different, the mixing mechanism is similar.

\footnotetext{
${ }^{\mathrm{a}}$ Corresponding author: mlkvik@fme.vutbr.cz
} 
Table 1. Important geometrical parameters of investigated atomizers

\begin{tabular}{|c|c|c|c|c|}
\hline & $D_{l}$ & $D_{0}$ & $\Theta$ & $N$ \\
\hline Y- jet & 0.4 & 1 & 52 & 1 \\
\hline OUIL & 1 & 9 & 90 & 20 \\
\hline
\end{tabular}

${ }^{*} D_{l}[\mathrm{~mm}]$ - liquid port diameter, $D_{0}[\mathrm{~mm}]-$ mixing chamber diameter, $\Theta\left[{ }^{\circ}\right]$ - injecting angle, $N$ - number of liquid ports

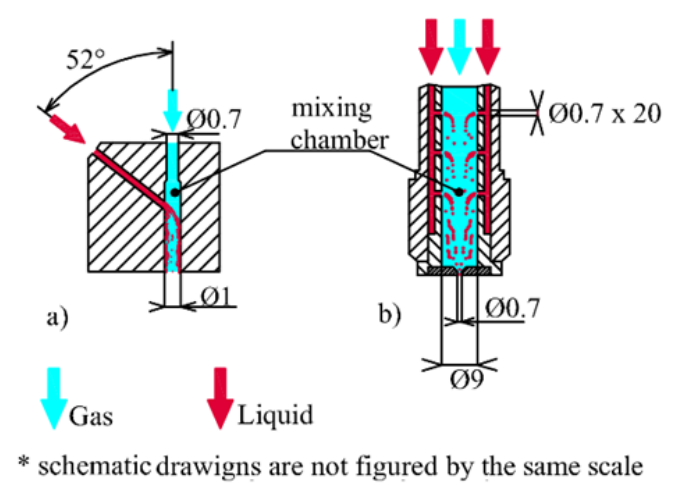

Figure 1. Schematic drawings of tested atomizers a) Y-jet, b) OUIL atomizer.

\section{The experiment}

An eccentric screw pump at constant rotation speed was used to drive the liquid into the atomizer. The volume flow rate towards the atomizer was adjusted using a needle valve. To provide the atomizer with atomization gas, compressed air from a house supply was used and adjusted by a pressure reducing valve. After ejection, the spray passed the measurement zone and was collected in a vessel which was further connected to an exhaust air fan. Inside the vessel a filter with large pores was used to prevent small spray drops from recycling into the measurement zone. The volume flow rate towards the atomizer was measured by a flow meter (VSE GmbH, Neuenrande, Germany). The gas pressure was measured by a pressure gauge shortly upstream of the atomizer. A thermal gas mass flow controller (High-Tech EL-Flow, Bronkhorst Mättig GmbH, Kamen, Germany) was used to indicate the resulting gas mass flow. The experiments were performed with three water solutions of the maltodextrine. The viscosities of the solutions were 147 and $60 \mathrm{mPa} \cdot \mathrm{s}$. The surface tensions of the solutions were 74.25 and $75.73 \mathrm{mN} / \mathrm{m}$.

\subsection{Measurements}

The external flow was observed using a high-speed camera (OLYMPUS i-speed2) at the frequency $10 \mathrm{kfps}$. The measuring volume was illuminated by a continuous LED lamp in a backlight configuration. A light diffuser was used to obtain uniform image background. The exposition time was set to $5 \mathrm{~ms}$. The PENTAX TV lens
(50 mm, fl:1.4) was used as a focusing optics. For the image magnification, the extension rings of a total length $25 \mathrm{~mm}$ were used. Due to the test rig construction, the output nozzle is not directly visible. The atomizer body allows us to observe the flow in the closest distance $3 \mathrm{~mm}$ downstream the output nozzle.

To enhance the image quality, the background was removed by subtracting the background image captured when no liquid was present. When the background is removed, the resultant images show only the liquid spray which appears as the grey or the black spots. The raw images shown a visible region of $6.3 \times 4 \mathrm{~mm}$. These images contained the part of the atomizer body, which appears as a black area in the right side of the image (Figure 2). Thus the visible area was cropped to the region of $5.3 \times 4 \mathrm{~mm}$.

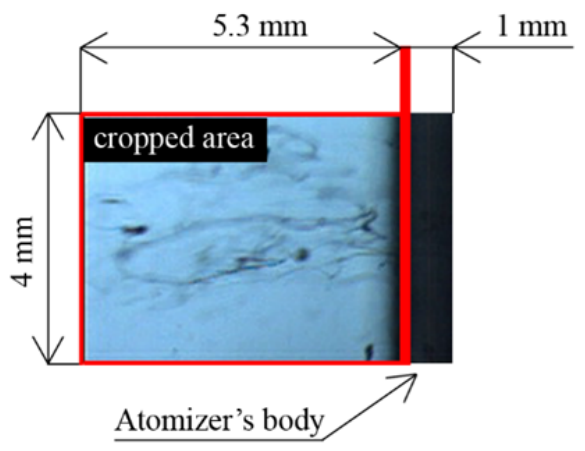

Figure 2. Cropping of the raw images.

\section{Considerations about internal two- phase flow of examined atomizers}

When the mixing principle is neglected, the flow pattern can change, according to the GLR, from the bubbly flow (at low GLR) up to the dispersed flow (at high GLR). Song [8] showed, that for the Y- jet atomizer, the internal flow is influenced by the mixing mechanism. To investigate this influence, Song [8] used the gas to the liquid momentum ratio. Because the mixing mechanism of the OUIL atomizer is very similar to the Y-jet atomizer, the internal flow of both atomizers is evaluated in terms of $\Phi$. According to the Tables 1 and 2, the liquid to the gas momentum ratio is significantly lower for the OUIL atomizer. Due to the different geometry of the examined atomizers (Table 1.), is $\Phi_{\text {OUIL }}$ approximately two orders lower than $\Phi_{\mathrm{Y}}$ for the same working regimes. According to the work of Song [8], when $\Phi<1$, the momentum of the liquid is low and the liquid stream forms a film on the wall at the liquid port side. When $1<\Phi<3$, the liquid penetrates into the center portion of the mixing chamber. For $\Phi>3$, the liquid can reach the wall opposite to the liquid port and forms there the liquid film (similar as in the first case). For the OUIL atomizer, $\Phi_{\text {OUIL }}$ remains very low (lower than 1). Hence, the momentum of the liquid cannot overcome the momentum of the gas and we presume that the liquid flows along the wall of the mixing chamber. As the liquid ports are placed symmetrically we can also expect the symmetric 
liquid film. Because each row of the liquid ports increases the flow rate in the liquid layer, we can presume, that this layer has a conical shape as shown in Figure 3.

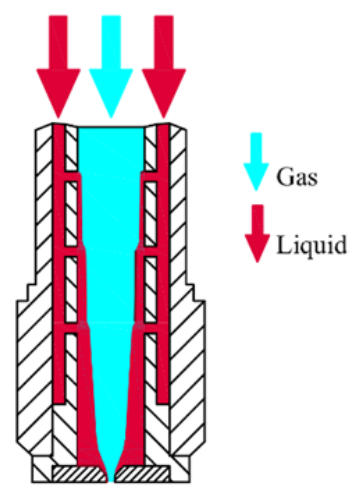

Figure 3. Presumed two-phase flow pattern in OUIL atomizer.

Table 2. Comparison of $\Phi$ at pressure level $0.14 \mathrm{MPa}$

\begin{tabular}{|c|c|c|c|c|c|}
\hline & \multirow{4}{*}{1} & \multirow{2}{*}{\multicolumn{2}{|c|}{ Y-jet }} & & \\
\hline & & & & \multicolumn{2}{|c|}{ OUIL } \\
\hline & & \multicolumn{2}{|c|}{$\mu \quad[\mathrm{mPa} \cdot \mathrm{s}]$} & \multicolumn{2}{|c|}{$\mu \quad[m P a \cdot s]$} \\
\hline & & 147 & 308 & 147 & 308 \\
\hline \multirow{4}{*}{$\frac{\sqrt{0}}{0}$} & 2.5 & 8.81 & 5.35 & 0.36 & 0.43 \\
\hline & 5 & 2.12 & 1.80 & 0.08 & 0.09 \\
\hline & 10 & 0.552 & 0.677 & 0.023 & 0.027 \\
\hline & 20 & 0.138 & 0.264 & 0.006 & 0.007 \\
\hline
\end{tabular}

Table 3. Comparison of $\Phi$ at pressure level $0.28 \mathrm{MPa}$

\begin{tabular}{|c|c|c|c|c|c|}
\hline & & \multicolumn{2}{|c|}{ Y-jet } & \multicolumn{2}{|c|}{ OUIL } \\
\hline & & \multicolumn{2}{|c|}{$\mu \quad[m P a \cdot s]$} & \multicolumn{2}{|c|}{$\mu[m P a \cdot s]$} \\
\hline & & 147 & 308 & 147 & 308 \\
\hline \multirow{4}{*}{$\frac{\sqrt{0}}{0}$} & 2.5 & 13.49 & 20.65 & 0.55 & 0.84 \\
\hline & 5 & 3.68 & 5.30 & 0.15 & 0.19 \\
\hline & 10 & 0.97 & 1.11 & 0.04 & 0.05 \\
\hline & 20 & 0.27 & 0.30 & 0.01 & 0.01 \\
\hline
\end{tabular}

If we consider the annular flow in the mixing chamber, we must also consider the stability of a liquid surface. The liquid surface becomes unstable, when the Bernoulli's force [2] overcomes the stabilizing influence of the surface tension $(\sigma)$. The stability of the liquid surface can be evaluated by the value of the critical wavelength (Eq. 1) [2]. This value defines the maximum stable wavelength of the surface disturbances. The $w$ in this equation denotes the gas to the liquid velocity slip.

$$
\lambda_{c}=2 \cdot \pi \cdot \sigma / \rho_{g} \cdot(w)^{2}
$$

For the Y-jet atomizer, we can expect the velocity of the gas in the mixing chamber within the range $12-$ $40 \mathrm{~m} / \mathrm{s}$ for all investigated working regimes (according to the measured flow rates). This gives the range of $\lambda_{c}$ within $0.1-2 \mathrm{~mm}$ for all of the operating regimes. As the expected gas velocity in the mixing chamber of the OUIL atomizer is in the range $0.3-0.6 \mathrm{~m} / \mathrm{s}$, the critical wavelength is considerably higher $(400-3000 \mathrm{~mm})$. This indicates that for the $\mathrm{Y}$ - jet atomizer, even disturbance of short wavelength becomes unstable and thus the liquid is torn apart into the small droplets which are carried in the gas core. On the other hand, the value of $\lambda_{c}$ for the OUIL atomizer exceeds the length of the mixing 10 to 100 times. Thus the probability of the unstable disturbance is very low and the gas core without a significant amount of droplets is expected.

\section{External flow}

The flow patterns, captured by the high speed camera, are shown in the Figures 5 and 6 . The regimes, where the atomizers did not produced the spray are denoted by the " $X$ " symbol. No images were captured for these regimes. The typical observed shape of the liquid structures is a fiber (ligament). The relative importance of the drag force, the viscosity and the surface tension is evaluated using the parameter $w \cdot \mu / \sigma$ (Figure 4 ). The high values of this parameter indicates, that the relative importance of $\sigma$ is low for all investigated regimes. At these conditions, the liquid deformation is driven by the drag force, while the liquid viscosity damps the deformation. As the result, deformation time is longer so the long ligaments are present in further distance from the output orifice [4].

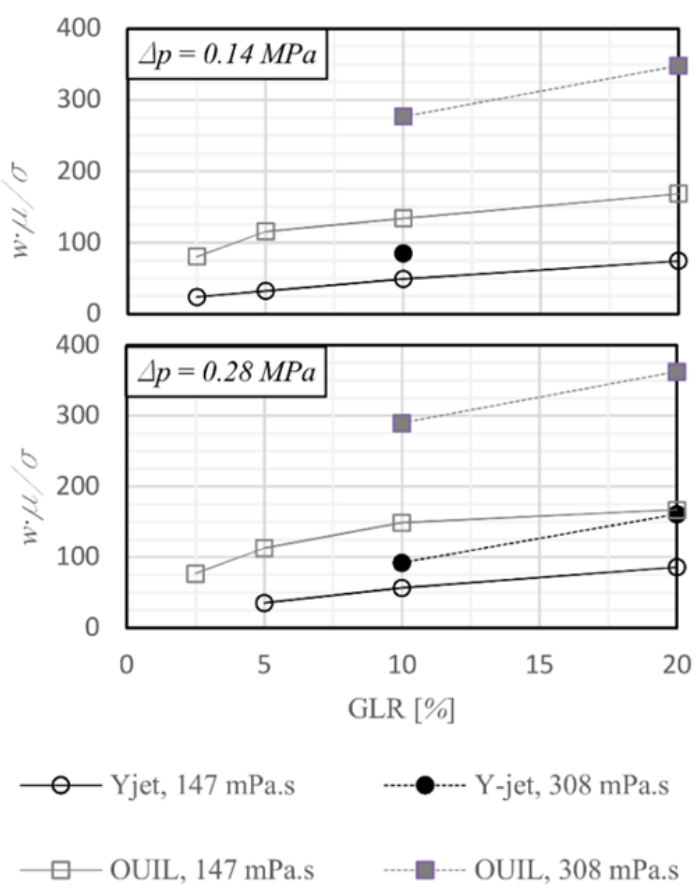

Figure 4. Relative importance of drag force, $\mu$ and $\sigma$.

The influence of the liquid surface stability can be observed in a morphology of the produced ligaments. Generally, the Y-jet atomizer produces the smooth ligaments. On the other hand, the ligaments produced by the OUIL atomizer are more branched with high amount of small structures connected to their surface. It can be expected, that the gas to the liquid interface is influenced by the instabilities, similar as in the case of internal flow 
(see section 4). Because the diameter of the output orifice of the OUIL atomizer is only $0.7 \mathrm{~mm}$, gas velocity in range within $50-100 \mathrm{~m} / \mathrm{s}$ can be expected when the gas expands. This value is based on the measured mass flowrates, when the pressure just behind the output orifice is considered as the atmospheric pressure. The Y-jet atomizer has the same diameter of the output orifice as the diameter of mixing chamber $(1 \mathrm{~mm})$, thus the gas velocity in range $12-40 \mathrm{~m} / \mathrm{s}$ is expected. The $\lambda_{c}$ of external flow for $\mathrm{Y}$-jet is in the range $0.3-3 \mathrm{~mm}$ and for the OUIL in range $0.04-0.2 \mathrm{~mm}$. The considerably lower value of $\lambda_{c}$ for the OUIL atomizer causes that smaller disturbances on the ligaments surface become unstable. This leads to the formation of small structures and consequently to the breakup of these structures into the small droplets. The values of $\lambda_{c}$ for the Y-jet atomizer are comparable to the ligament length, especially for the lower pressure. As the pressure drop increases $(0.28 \mathrm{MPa})$, the value of $\lambda_{c}$ becomes smaller which leads to the formation of highly branched ligaments. This effect can be observed especially for the liquid of higher viscosity, where the breakup process is slow and long, branched ligaments are present.

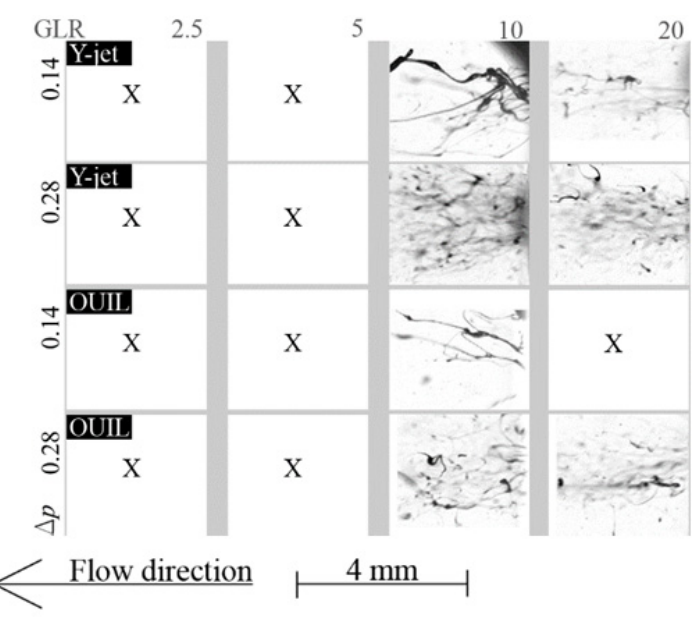

Figure 5. Spray pattern for liquid viscosity $308 \mathrm{mPa} \cdot \mathrm{s}$, GLR [\%], $\triangle p[M P a]$.

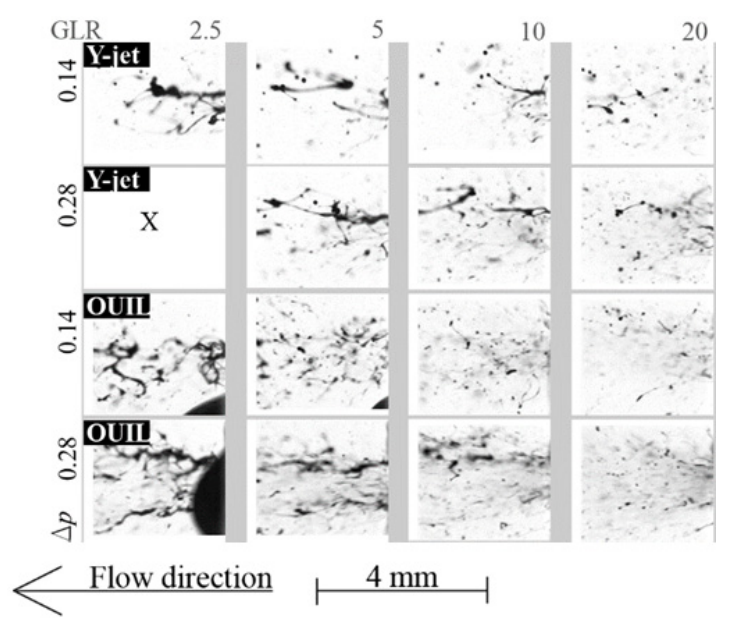

Figure 6. Spray pattern for liquid viscosity $147 \mathrm{mPa}$, GLR [\%], $\triangle p[M P a]$.

\section{Conclusions}

The spray, generated by the Y- jet atomizer and the OUIL atomizer, was examined. The design and mixing mechanism of these atomizers are described in the section 2.1. The internal multiphase flow is evaluated in terms of the liquid to the gas momentum ratio, which is the criterion used for estimating the internal flow, influenced by the mixing mechanism, in Y-jet atomizers [8]. Different types of the internal flow pattern were estimated for the $\mathrm{Y}$ - jet atomizer. The values of $\Phi_{\mathrm{Y}}$, from 20 to 0.3 , and very low $\lambda_{c}$ (from 0.1 to $2 \mathrm{~mm}$ ) indicates the intensive gas to the liquid interaction and presence of the small droplets in the gas core in the mixing chamber of the $\mathrm{Y}$ - jet atomizer. For the OUIL atomizer $\Phi_{\text {OUIL }}<1$ and $\lambda_{c}$ is comparable to the length of the mixing chamber, which indicates the annular flow and the high level of flow separation (Figure 3). The relative comparison of the basic forces affecting external flow (Figure 4) indicates, that the liquid deformation process was influenced mainly by the drag force and damping viscous force, which leads to presence of the long ligaments (Figure 5 and 6). The higher gas to the liquid velocity slip leads to the branching and faster disruption of ligaments in case of the OUIL atomizer. Consequently, when fast atomization is the priority (under the same working parameters), the OUIL atomizer seems to be a better choice for atomization of the examined viscous liquids.

\section{Acknowledgement}

Authors acknowledge financial support from project № 101/11/1264 funded by the Czech Grant Agency, from project NETME CENTRE PLUS (LO1202) co-funded by the Ministry of Education, Youth and Sports within the support programme „National Sustainability Programme I“ and project № CZ.1.07/2.3.00/30.0005 of Operational Program Education for Competitiveness of Ministry of Education, Youth and Sport of the Czech Republic.

\section{References}

1. L.P. Bayvel, Z. Orzechowski, Liquid atomization (Taylor \& Francis, Washington, 1993)

2. C. E. Brennen, Fundamentals of two-phase flows (Cambridge university press, 2005)

3. H. P. Gadgil, B. N. Raghunandan, Exp. Fluids (2011)

4. L. P. Hsiang, Int. J. Multiphase Flow, (1995)

5. J. Jedelsky, M. Jícha, Fuel 111 (2013)

6. R. Olsiak, B. Knizat, M. Mlkvik, Komunikacie, 14 (4 A), pp. 58-62

7. P. J. Mullinger, N.A. Chigier, J. Inst. Fuel 47 (1974)

8. S. H. Song, S. Y. Lee, Atomization and sprays vol. 6 (1993)

9. S. Sovani, P. Sojka, A. Lefebvre, Prog. Energ. Combust. 27(4), (2001) 